\title{
Oligomer Formation in Evaporating Aqueous Glyoxal and Methyl Glyoxal Solutions
}

Kirsten W. Loeffler, Charles A. Koehler, Nichole M. Paul, and David O. De Haan*

Chemistry Department, University of San Diego, 5998 Alcala Park, San Diego CA 92110

*corresponding author. (619) 260-6882. Fax: (619) 260-2211. e-mail: ddehaan@sandiego.edu 
Supporting Information

The effects of glyoxal concentration on the ATR spectra of the dried residue are shown in Figure A. These spectra have been scaled and offset to allow peak shapes to be compared. It is apparent that the 1 and $50 \mathrm{mM}$ runs have almost identical peak shapes, while the $1 \mathrm{M}$ run (top) has a stronger peak at $950 \mathrm{~cm}^{-1}$, which we attribute to the asymmetrical $\mathrm{C}-\mathrm{O}$ stretching vibration of dioxolane rings. The relative peak sizes in the $1 \mathrm{M}$ glyoxal experiment are very similar to that of the solid glyoxal trimer dihydrate standard, shown in Fig.1.

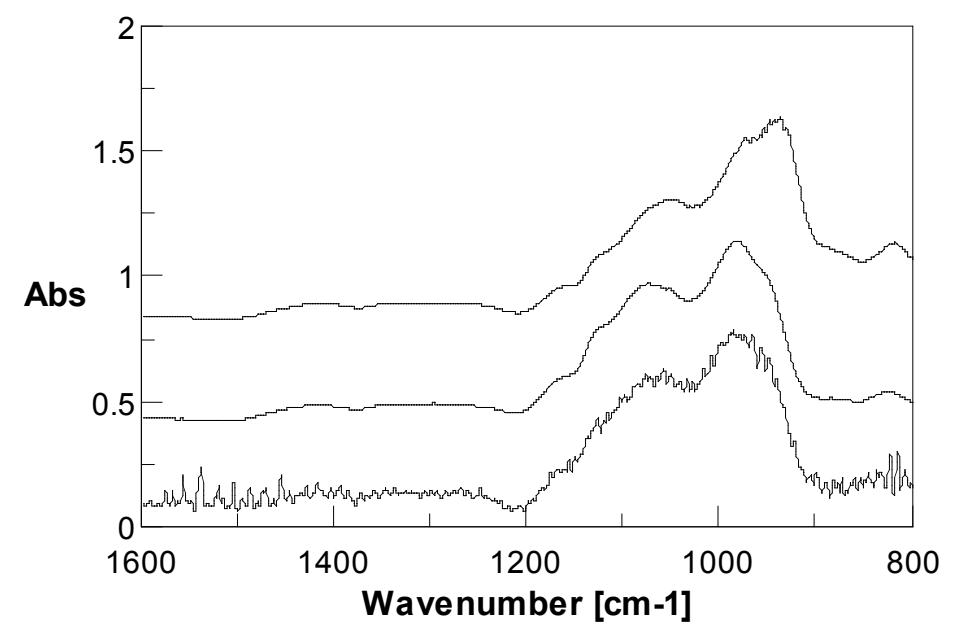

Figure A: C-O stretching bands of final attenuated total reflectance FTIR spectra of glyoxal drying experiments at difference starting concentrations. Spectra are scaled for comparison of peak shapes. Top: $1.0 \mathrm{M}$ glyoxal solution (scaled by a factor of 0.44 and offset by +0.80 absorbance units). Middle: $0.050 \mathrm{M}$ glyoxal solution (offset by +0.40). Bottom: 0.0010 M glyoxal solution (scaled by 24.4 and offset by +0.40 ). The experiments were performed using 1 $\mu \mathrm{L}$ droplets. 
The dependence of glyoxal oligomer formation on evaporation rate was examined first by varying the ratio of acetone to water in the solvent. Sample data is overlaid in Figure B, scaled to show similarity of peaks. This similarity indicates a similar extent of glyoxal oligomer formation in each experiment. Peak intensity varies from run to run due to the random location of the evaporated residue on the ATR crystal surface. There was no trend overall between either relative or absolute peak intensity and acetone/water solvent ratios.

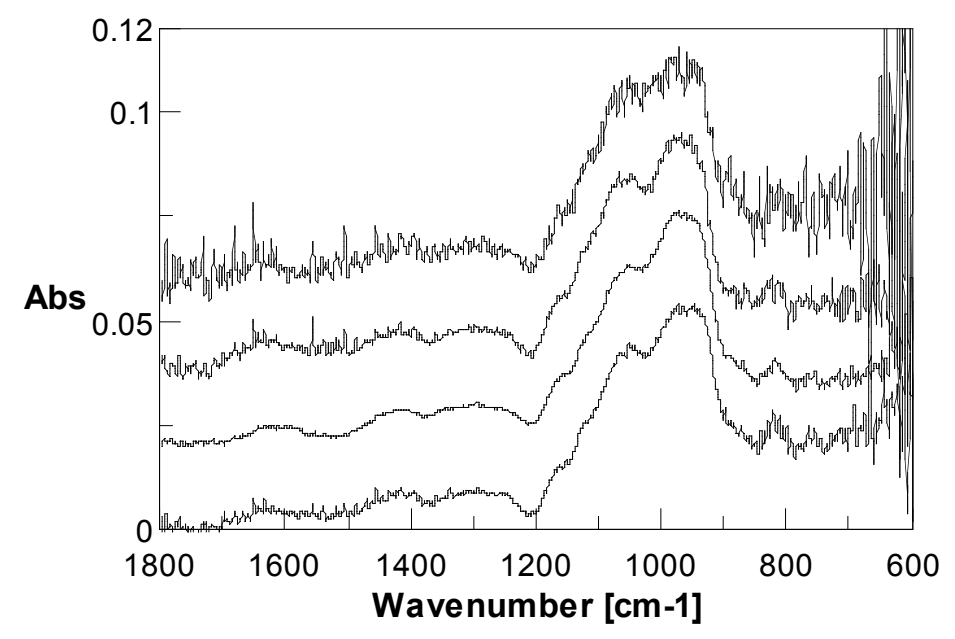

Figure B: ATR spectra of dried glyoxal residue, from $1 \mu \mathrm{L}$ droplets of $10 \mathrm{mM}$ glyoxal solutions with varying acetone / water ratios. Top: $75 \%$ acetone (scaled by 2.5 and offset by +0.06 ). $2^{\text {nd. }}$ : $50 \%$ acetone (offset by +0.04$) .3^{\text {rd }}: 10 \%$ acetone (scaled by 0.10 and offset by +0.02 ). Bottom: No acetone present.

The dependence of glyoxal oligomer formation on droplet size is shown in Figure C, again scaled to allow the comparison of peak shapes. This similarity shows that oligomer formation is not dependent on the size of the droplet that is dried within the experimental range of 0.25 to 5 $\mu \mathrm{L}$. (In general, signals were weaker with larger drop sizes. This is due to the heterogeneity of coverage of dried residue on the ATR crystal surface, and the greater likelihood with large drop sizes of dried residues locating further from the center of the ATR crystal.) 


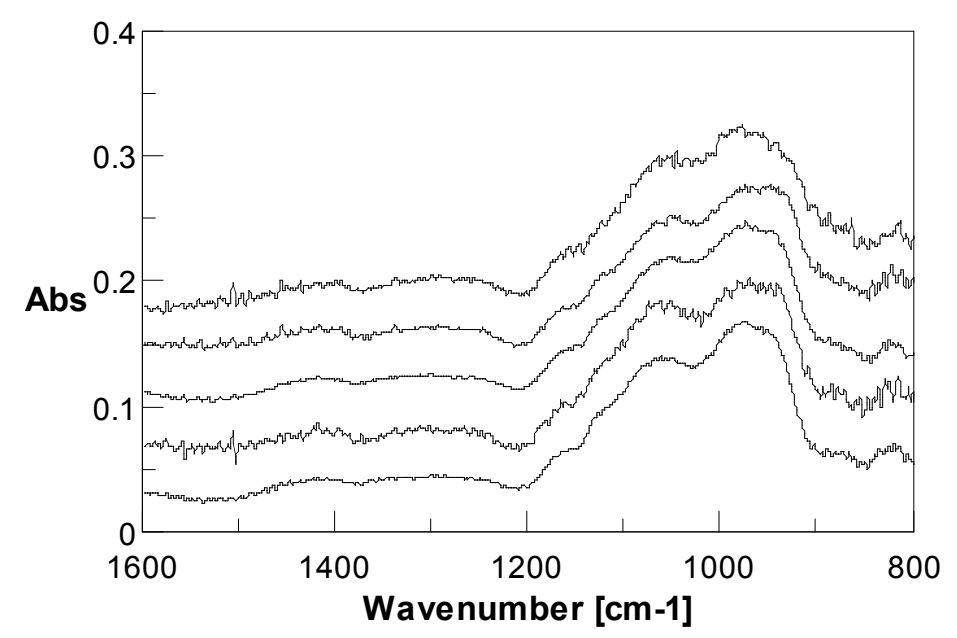

Figure C: ATR spectra of dried glyoxal residue, from $10 \mathrm{mM}$ glyoxal solutions of varying drop sizes. Top: $5 \mu \mathrm{L}$ droplet (scaled by 3.952 and offset by +0.18 ). $2^{\text {nd }}$ from top: $3 \mu \mathrm{L}$ droplet (scaled by 2.544 and offset by +0.14 ). Middle: $1 \mu \mathrm{L}$ droplet (scaled by 1.26 and offset by +0.10 ). $4^{\text {th }}$ from top: $0.5 \mu \mathrm{L}$ droplet (scaled by 4.795 and offset by +0.06 ). Bottom: $0.25 \mu \mathrm{L}$ droplet (scaled by 1.981 and offset by +0.02 ).

Spectral are shown for $10 \mathrm{mM}$ glyoxal droplets drying at various relative humidity levels in Figure D. The ambient relative humidity for runs performed without a cover is $\sim 35 \% \mathrm{RH}$ (bottom spectrum). In other runs, humidity was raised by placing a cover over the ATR surface, adding pre-mixed flows of humidified and dried nitrogen, and monitoring humidity levels with a hygrometer. Although evaporation rates are slowed at high humidity levels, no systematic changes in either peak shape or peak intensity were observed in response to varying humidity between 35 and 98\% RH. 


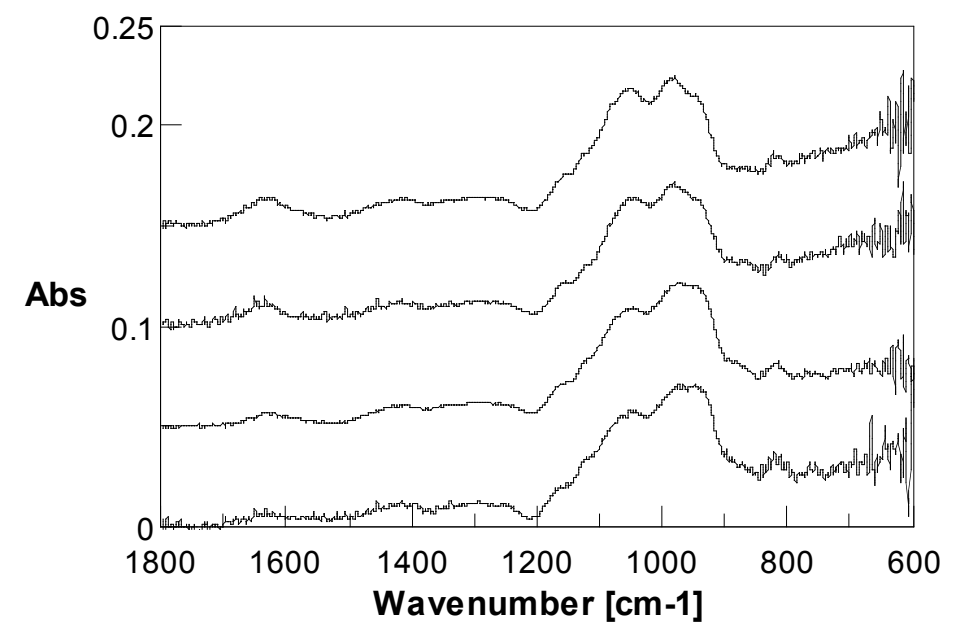

Figure D: ATR spectra of the dried residue of $1 \mu \mathrm{L}$ droplets of $10 \mathrm{mM}$ glyoxal solution in water, dried at various relative humidity levels. Top: $98 \% \mathrm{RH}$ (offset by +0.15 ). $2^{\text {nd }}$ from top: $70 \% \mathrm{RH}$ (offset by +0.1$) .3^{\text {rd }}: 57 \%$ RH (offset by +0.05 ). Bottom: $\sim 35 \%$ RH (scaled by a factor of 1.33 , initial drop size $3 \mu \mathrm{L})$.

The lack of dependence on relative humidity is confirmed by mass measurements of dried residues of arrays of larger, more concentrated glyoxal droplets (Figure E). Glyoxal recoveries in the dried residue are $100 \%$ (within experimental error). On the other hand, methyl glyoxal recoveries are $100 \%$ only up to $70 \% \mathrm{RH}$. At $95 \% \mathrm{RH}$, a substantial mass deficit due to methyl glyoxal evaporation was recorded. 


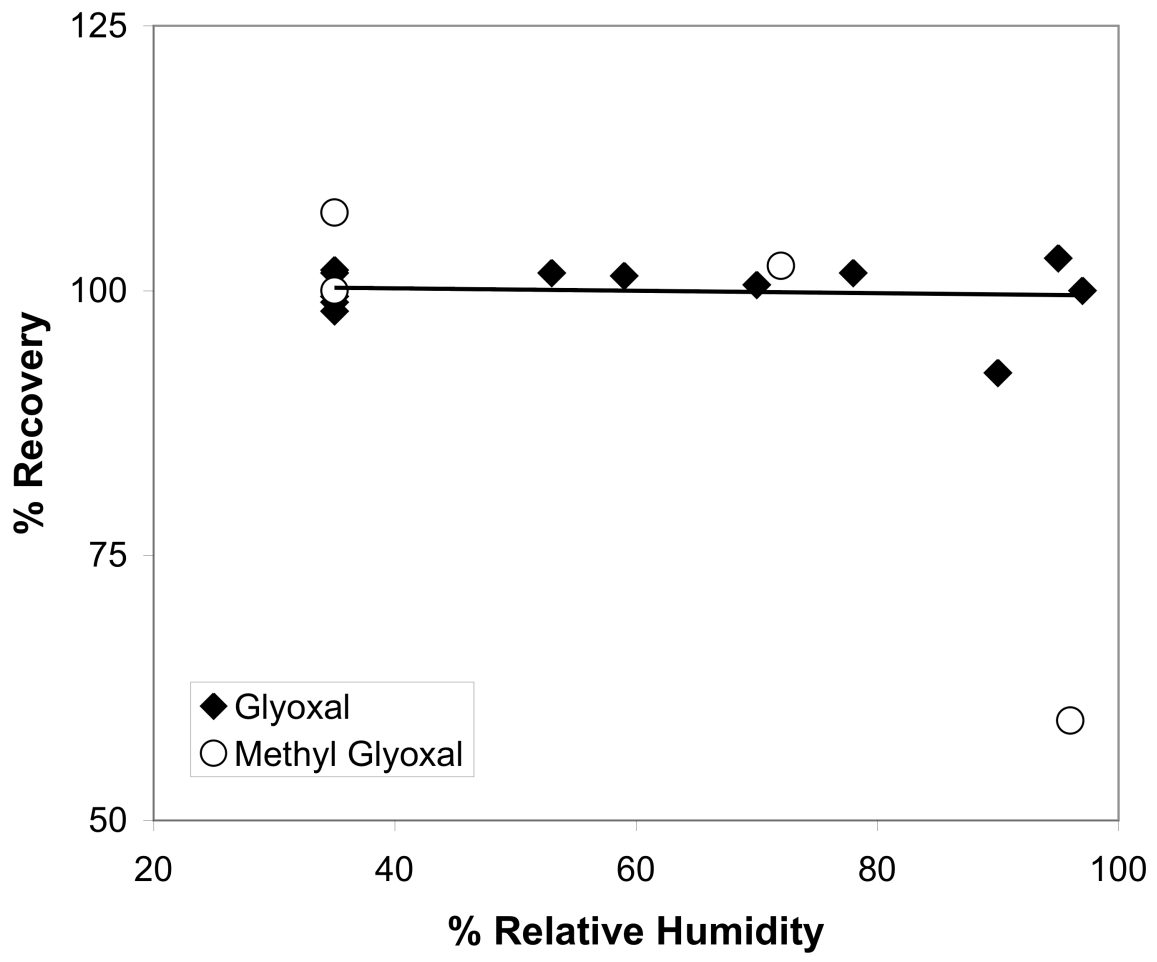

Figure E: Mass-based \% recovery results from evaporation of twenty $25 \mu \mathrm{L}$ droplets of $1.0 \mathrm{M}$ glyoxal or methyl glyoxal at various relative humidity levels. Recovery is based on the expected formation of glyoxal trimer dihydrate or methyl glyoxal monohydrate, respectively. The main source of uncertainty is the $\pm 3 \%$ precision of the autopipette used for droplet delivery.

Spectral overlays for experiments where solutions containing both $10 \mathrm{mM}$ glyoxal and $10 \mathrm{mM}$ organic acids were dried on the ATR crystal are shown in Figures F, G and H. Some spectral interference in the $\mathrm{C}-\mathrm{O}$ stretch region is seen in all figures. After deconvolution, glyoxal oligomer formation appeared to be accelerated beyond glyoxal-only experiments only in the presence of glyoxylic acid, Fig. H. 


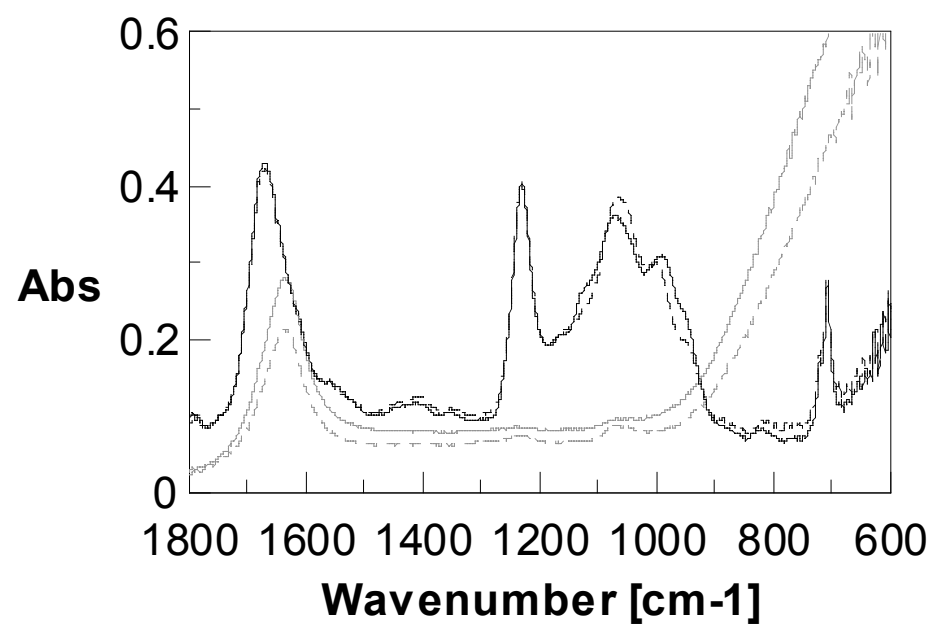

Figure F: $10 \mathrm{mM}$ Glyoxal $+10 \mathrm{mM}$ oxalic acid solution drying on ATR diamond crystal at $22 \mathrm{C}$ and $35 \%$ RH. 2.2 min: Gray solid line. 3.3 min: Gray dash. 4.4 min: black dash. 14.6 min: black solid. Peaks at 1230 and $1670 \mathrm{~cm}^{-1}$ in the dried residue (black) are due to oxalic acid. Oxalic acid also contributes slightly to the peak at $1070 \mathrm{~cm}^{-1}$.

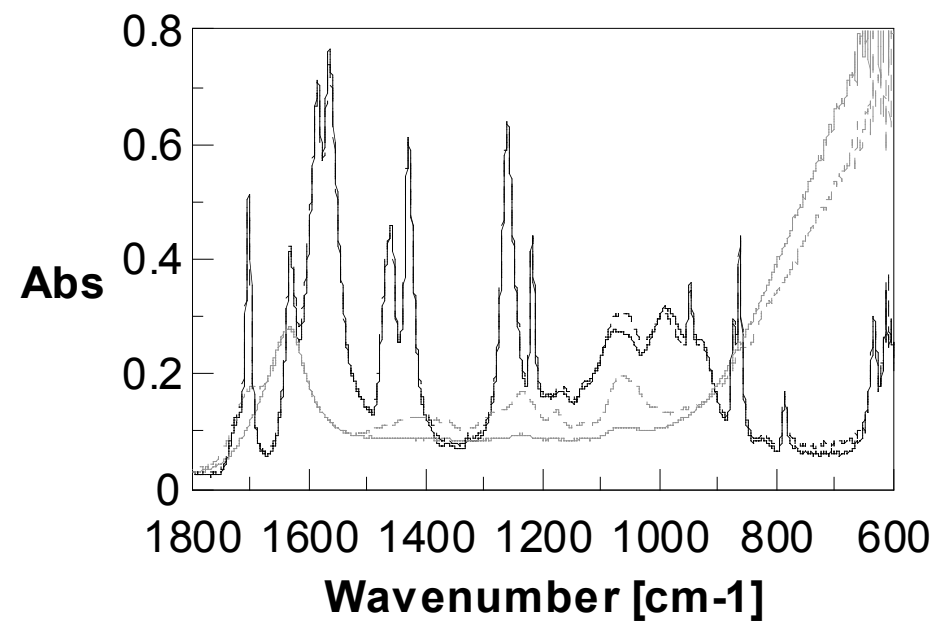

Figure G: $10 \mathrm{mM}$ Glyoxal $+10 \mathrm{mM}$ maleic acid solution drying on ATR diamond crystal at 22 C and $35 \%$ RH. 4.1 min: Gray solid line. 5.2 min: Gray dash. 6.3 min: black dash. 15 min: black solid. Narrow peaks at 948 and $864 \mathrm{~cm}^{-1}$ and all peaks to the left of $1200 \mathrm{~cm}^{-1}$ are due to maleic acid in the scans of the dried residue (black). 


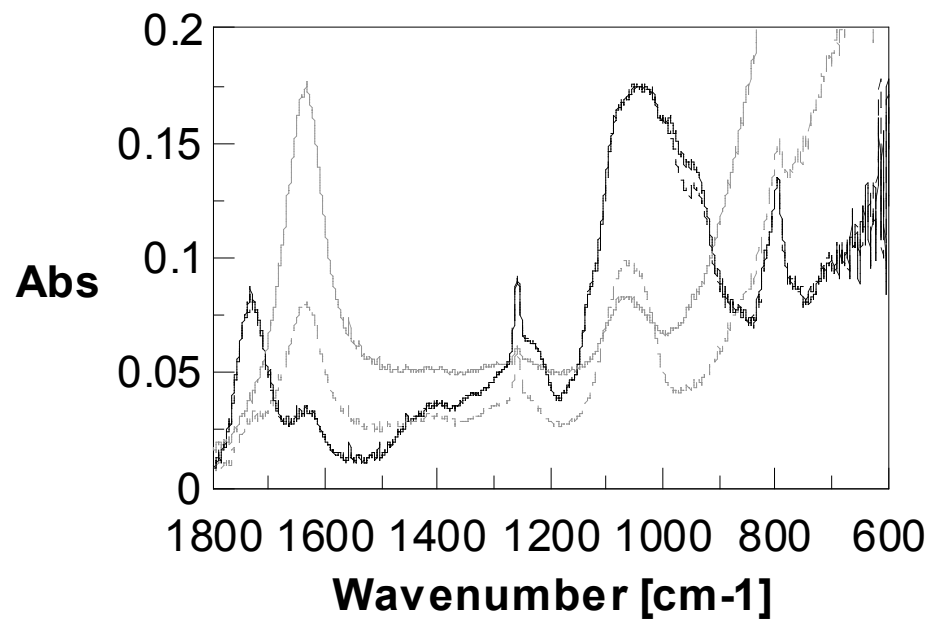

Figure H: $10 \mathrm{mM}$ Glyoxal $+10 \mathrm{mM}$ glyoxylic acid solution drying on ATR diamond crystal at $22 \mathrm{C}$ and $35 \%$ RH. 7.4 min: Gray solid line. 8.6 min: Gray dash. 10.0 min: black dash. 15.4 min: black solid. Peaks at 1730, 1260 and $800 \mathrm{~cm}^{-1}$ are due to glyoxylic acid in the scans of the dried residue (black). Glyoxylic acid also contributes substantially to the broad peak at $1050 \mathrm{~cm}^{-}$ ${ }^{1}$, but much less so at 980 and $950 \mathrm{~cm}^{-1}$, making deconvolution of the signals for glyoxylic acid and glyoxal possible. 